MATHEMATICS OF COMPUTATION

Volume 73 , Number 246 , Pages $843-851$

S 0025-5718(03)01539-4

Article electronically published on September 2, 2003

\title{
A SENSITIVE ALGORITHM FOR DETECTING THE INEQUIVALENCE OF HADAMARD MATRICES
}

\author{
KAI-TAI FANG AND GENNIAN GE
}

\begin{abstract}
A Hadamard matrix of side $n$ is an $n \times n$ matrix with every entry either 1 or -1 , which satisfies $H H^{T}=n I$. Two Hadamard matrices are called equivalent if one can be obtained from the other by some sequence of row and column permutations and negations. To identify the equivalence of two Hadamard matrices by a complete search is known to be an NP hard problem when $n$ increases. In this paper, a new algorithm for detecting inequivalence of two Hadamard matrices is proposed, which is more sensitive than those known in the literature and which has a close relation with several measures of uniformity. As an application, we apply the new algorithm to verify the inequivalence of the known 60 inequivalent Hadamard matrices of order 24; furthermore, we show that there are at least 382 pairwise inequivalent Hadamard matrices of order 36 . The latter is a new discovery.
\end{abstract}

\section{INTRODUCTION}

A Hadamard matrix of side $n$ is an $n \times n$ matrix with every entry either 1 or -1 , which satisfies $H H^{T}=n I$. It is known that $n$ is necessarily 1,2 , or a multiple of four. A Hadamard matrix is called standardized if its first row and column consist of all 1's. Two Hadamard matrices are called equivalent if one can be obtained from the other by some sequence of row and column permutations and negations. The equivalence classes of Hadamard matrices of order $\leq 28$ have been obtained by Hall [7], 8], Ito et al. [10], Kimura [11, [12, [13] and Spence [20]. On the equivalence class of Hadamard matrices, we have the following known results (see 2, Theorem 24.34], 21], [6]).

Lemma 1.1. There is a unique equivalence class of Hadamard matrices of each order 1,2,4,8, and 12. The number of classes for orders $16,20,24,28,32$, and 36 are $5,3,60,487, \geq 66000$, and $\geq 200$, respectively.

For identifying the equivalence of two Hadamard matrices of order $n$, a complete search compares $\left(2^{n} n !\right)^{2}$ pairs of matrices and is known to be an NP hard problem when $n$ increases. Kimura used the K-matrix for classification of the Hadamard matrices of order 28. For the definition of $K$-matrix associated with Hadamard

Received by the editor July 9, 2001 and, in revised form, November 28, 2001.

2000 Mathematics Subject Classification. Primary 68Q15, 05B20, 62K15.

Key words and phrases. Algorithm, equivalence, Hadamard matrix, Hamming distance, uniformity.

This research was supported in part by the Hong Kong RGC grants HKBU RC/98-99/Gen370 and HKBU 2044/02P. The second author was also supported by statistics Research and Consultancy Centre, Hong Kong Baptist University and the YNSFC Grant 10001026. 
matrices, see 14. Smith normal form can certainly be applied as an invariable for equivalent Hadamard matrices (see [4]), which is easy to compute but very coarse for orders above 16. Several authors (see [3], [15], [16], [17]) proposed using the profile to identify the inequivalence of two Hadamard matrices, which is more sensitive than Smith normal form.

Suppose that $H=\left(h_{i j}\right)$ is a Hadamard matrix of order $n \geq 8$. Define

$$
p_{i j k l}=\left|\sum_{x=1}^{n} h_{i x} h_{j x} h_{k x} h_{l x}\right| .
$$

Denote by $\pi(m)$ the number of sets $\{i, j, k, l\}$ of four distinct rows such that $p_{i j k l}=$ $m$. We call $\pi(m)$ the 4-profile of $H$. Similarly, we can define the 6 -profile, 8 -profile, etc. For some modified version of the profile, such as the extended profile and the generalized profile, can refer to [16. Two equivalent Hadamard matrices have the same profile, but the inverse is not true. We shall see that the profile criterion is not sensitive enough to detect inequivalent Hadamard matrices. Therefore, we need some criterion that is more sensitive than the profile and that is easy to compute. Recently Ma, Fang and Lin [18] suggested using the uniformity and projection uniformity that have been used in quasi-Monte Carlo methods (Hickernell [9] and Niederreiter [19]) to detect nonisomorphism of factorial designs. Their idea may be useful in detecting inequivalent Hadamard matrices. Because isomorphism in factorial designs and equivalence in Hadamard matrices have different definitions, we have to find a new criterion for Hadamard matrices.

The main purpose of this paper is to propose a new criterion that is based on the symmetric Hamming distances of the rows of a Hadamard matrix and their projections. The symmetric Hamming distance is a modified version of Hamming distance and will be defined in the next section. The new criterion has a close link with several measures of uniformity. We shall show that two Hadamard matrices are equivalent if the Hamming distances between the rows are the same in all possible projections. A new algorithm for detecting inequivalence of two Hadamard matrices is proposed in Section 3. As an application of the new criterion and algorithm, we will verify the inequivalence of the known 60 inequivalent Hadamard matrices of order 24 in Section 4, and we will show in Section 5 that there are at least 382 inequivalent Hadamard matrices of order 36. A connection of the new criterion and measure of uniformity, such as the centered $L_{2}$-discrepancy that has been used in quasi-Monte Carlo methods as well as in factorial designs, will be pointed out in the last section.

\section{A NECESSARY AND SUFFICIENT CONDITION}

Suppose that $H$ is a Hadamard matrix of side $n$. Let $D_{H}=\left(d_{i j}\right)$ be the Hamming distance matrix of a Hadamard matrix $H$, where $d_{i j}$ is the Hamming distance of the $i$-th and $j$-th rows of $H$ and is defined as the number of positions with different elements.

Recently, Clark and Dean in [1, Theorem 2.1] presented a necessary and sufficient condition for the equivalence of two fractional factorial designs in terms of Hamming distance matrices. Since a Hadamard matrix can be regarded as a fractional factorial design, we have the following sufficient condition for Hadamard matrices.

Theorem 2.1. Let $\left\{c_{1}, \ldots, c_{n}\right\}$ be any permutation of the integers $\{1, \ldots, n\}$ and let $D_{H}\left(c_{1}, \ldots, c_{q}\right)$ be the distance matrix corresponding to columns $\left\{c_{1}, c_{2}, \ldots, c_{q}\right\}$ 
of $H$. For any given permutation $\left\{c_{1}, c_{2}, \ldots, c_{n}\right\}$ of $\{1,2, \ldots, n\}$ and any given $n \times n$ permutation matrix $R$, the sequence of matrices

$$
R D_{H}\left(c_{1}, c_{2}, \ldots, c_{q}\right) R^{\prime}, \quad q=1,2, \ldots, n,
$$

uniquely determines the Hadamard matrix $H$ up to equivalence.

Remark. The reverse of Theorem[2.1 is also true for fractional factorial designs, but it is not the case for Hadamard matrices. The main reason is that the definition of isomorphism for factorial designs and the definition of equivalence for Hadamard matrices are not identical. For factorial designs, the negation of a row is not allowed.

Corollary 2.2. Hadamard matrices $H_{1}$ and $H_{2}$ are equivalent if there exists an $n \times n$ permutation matrix $R$ and a permutation $\left\{c_{1}, c_{2}, \ldots, c_{n}\right\}$ of $\{1,2, \ldots, n\}$ such that, for $q=1,2, \ldots, n$,

$$
D_{H_{1}}(q)=R D_{H_{2}}\left(c_{q}\right) R^{\prime} .
$$

Let $H\left(c_{1}, \ldots, c_{q}\right)$ denote a matrix consisting of columns $c_{1}, \ldots, c_{q}$ of $H$. Note that $D_{H}\left(c_{1}, \ldots, c_{q}\right)=\left(q J_{q}-H\left(c_{1}, \ldots, c_{q}\right) H^{\prime}\left(c_{1}, \ldots, c_{q}\right)\right) / 2$, where $J_{q}$ is a $q \times q$ matrix of all ones. We then have the following corollary.

Corollary 2.3. Hadamard matrices $H_{1}$ and $H_{2}$ are equivalent if and only if there exists an $n \times n$ permutation matrix $R$ and a permutation $\left\{c_{1}, c_{2}, \ldots, c_{n}\right\}$ of $\{1,2, \ldots, n\}$ such that, for $q=1,2, \ldots, n$,

$$
H_{1}(1,2, \ldots, q) H_{1}{ }^{\prime}(1,2, \ldots, q)=R H_{2}\left(c_{1}, c_{2}, \ldots, c_{q}\right) H_{2}{ }^{\prime}\left(c_{1}, c_{2}, \ldots, c_{q}\right) R^{\prime} .
$$

For a Hadamard matrix $H$, the Hamming distance matrix $D_{H}$ is invariant only to permutations and negations on columns of $H$. Hence, we need a new conception on distance which is more suitable for the study of classification of Hadamard matrices.

Let $S D_{H}=\left(s_{i j}\right)$ be the symmetric Hamming distance matrix of a Hadamard matrix $H$, where $s_{i j}$ is the symmetric Hamming distance of the $i$-th and $j$-th rows of $H$ and is defined as the smaller number of positions with the same entries and different entries. For example, the Hamming distance and symmetric Hamming distance of two rows $(+,+,-,-,+,+,-,-)$ and $(+,-,+,-,-,-,+,+)$ are 6 and 2 , respectively.

Note that the symmetric Hamming distance matrix $S D_{H}$ is invariant to permutations of columns and to negations of both rows and columns of $H$. Hence, we have the following necessary condition for equivalence of Hadamard matrices.

Theorem 2.4. If Hadamard matrices $H_{1}$ and $H_{2}$ are equivalent, then there exists a permutation matrix $R$ such that

$$
S D_{H_{1}}=R\left(S D_{H_{2}}\right) R^{\prime},
$$

where $R^{\prime}$ denotes the transpose of $R$.

\section{An AlgORithm FOR the ineQuivalence of Hadamard Matrices}

In this section we consider the problem of detecting inequivalence for two Hadamard matrices. If we directly use the theory developed in the previous section, to identify two Hadamard matrices or to detect inequivalence of two Hadamard matrices is still an NP hard problem when $n$ increases. Therefore, we define a set of criteria that are functions of symmetric Hamming distances and projection symmetric Hamming distances of a Hadamard matrix. For any given Hadamard matrix $H$ of order $n$, let $S_{i}(H)$ be the number of pairs of two distinct rows whose 
symmetric Hamming distance is $i$. The sequence $\left(S_{0}(H), \ldots, S_{n / 2}(H)\right)$ is referred to as the distance distribution of $H$.

Denote

$$
B_{a}(H)=\sum_{i=0}^{n / 2} S_{i}(H)\left(a^{i}+a^{n-i}\right)
$$

as the distance enumerator of $H$, where $a$ is a positive number. Given $k(1 \leq k \leq n)$, the distribution of $B_{a}$-values over all $k$-dimensional column projections is denoted by $F_{B_{a, k}}(H)$. It is not difficult to see that $F_{B_{a, k}}(H)$ is invariant to row and column permutations and negations. Hence, we propose the following necessary condition for equivalence of Hadamard matrices.

Theorem 3.1. If Hadamard matrices $H_{1}$ and $H_{2}$ are equivalent, then $F_{B_{a, k}}\left(H_{1}\right)=$ $F_{B_{a, k}}\left(H_{2}\right)$ for $k=1,2, \ldots, n / 2$.

From Theorem 3.1 we can conclude that $H_{1}$ and $H_{2}$ are inequivalent if $F_{B_{a, k}}\left(H_{1}\right)$ $\neq F_{B_{a, k}}\left(H_{2}\right)$ for some $k$. The following two lemmas can save much computational work.

Lemma 3.2. The relations $F_{B_{a, k}}\left(H_{1}\right)=F_{B_{a, k}}\left(H_{2}\right), k=1,2$, hold for any given Hadamard matrices $H_{1}$ and $H_{2}$ with the same order.

Proof. Since any Hadamard matrix is equivalent to its standardized form, we can suppose that both of $H_{1}$ and $H_{2}$ are standardized. Note that any column of a standardized Hadamard matrix has half 1's and half -1 's. The assertion for $k=1$ follows. For the case of $k=2$, since any two columns of a Hadamard matrix are orthogonal, it is easy to see that their distance distributions are identical. Hence, $F_{B_{a, 2}}\left(H_{1}\right)=F_{B_{a, 2}}\left(H_{2}\right)$. This completes the proof.

Since any two rows of a Hadamard matrix of order $n$ are orthogonal, we have that the Hamming distance between them is $n / 2$. Note that the residue of a $k$ dimensional projection of $H$ corresponds to an $(n-k)$-dimensional projection of $H$ and vice versa. Hence, we have

Lemma 3.3. Let $H_{1}$ and $H_{2}$ be two Hadamard matrices of order $n$. For any $k$ $(1 \leq k \leq n)$,

$$
F_{B_{a, k}}\left(H_{1}\right)=F_{B_{a, k}}\left(H_{2}\right) \text { if and only if } F_{B_{a, n-k}}\left(H_{1}\right)=F_{B_{a, n-k}}\left(H_{2}\right) .
$$

Proof. Let $H^{k}$ denote the $k$-dimensional projection of a Hadamard matrix $H$ and let $d_{r_{1}, r_{2}}^{k}$ denote the Hamming distance of row $r_{1}$ and $r_{2}$ of $H^{k}$. For any two given rows $r_{1}$ and $r_{2}$ of $H_{1}{ }^{k}$, if their symmetric Hamming distance is equal to that of rows $r_{3}$ and $r_{4}$ of $H_{2}{ }^{k}$, then we have that $d_{r_{1}, r_{2}}^{k}=d_{r_{3}, r_{4}}^{k}$ or $d_{r_{1}, r_{2}}^{k}=k-d_{r_{3}, r_{4}}^{k}$. Since $d_{r_{1}, r_{2}}^{k}+d_{r_{1}, r_{2}}^{n-k}=\frac{n}{2}$, we have that $d_{r_{1}, r_{2}}^{n-k}=d_{r_{3}, r_{4}}^{n-k}$ or $d_{r_{1}, r_{2}}^{n-k}=(n-k)-d_{r_{3}, r_{4}}^{n-k}$. Hence, the symmetric Hamming distance of rows $r_{1}$ and $r_{2}$ of $H_{1}{ }^{n-k}$ is identical to that of rows $r_{3}$ and $r_{4}$ of $H_{2}{ }^{n-k}$. The assertion then follows.

From Theorem 3.1 and Lemmas 3.2 and 3.3 , we propose the following algorithm for detecting inequivalent Hadamard matrices, where the parameter $a$ is chosen to be an irrational number. 


\section{An algorithm for detecting inequivalent Hadamard matrices.}

Step 1. Let $k=3$.

Step 2. Compare $F_{B_{a, k}}\left(H_{1}\right)$ and $F_{B_{a, k}}\left(H_{2}\right)$ and check whether $F_{B_{a, k}}\left(H_{1}\right)=$ $F_{B_{a, k}}\left(H_{2}\right)$. If no, go to Step 4; otherwise go to Step 3.

Step 3. If $k=n / 2$, we fail to identify equivalence between $H_{1}$ and $H_{2}$ and we need further study; otherwise let $k:=k+1$ and go to Step 2.

Step 4. We conclude that $H_{1}$ and $H_{2}$ are not equivalent and terminate the process.

Remark. We can see that symmetric distance is closely related to inner product. Let $K$ be a $k$-subset of $X=\{1,2, \ldots, n\}$. Let $u_{K}, v_{K}$ be the $i$-th and $j$-th row of $H^{K}$, respectively. Denote

$$
\left\langle u_{K}, v_{K}\right\rangle=\sum_{c \in K} h_{i c} h_{j c}
$$

Let $d$ be the symmetric distance of $u_{K}$ and $v_{K}$. When $d$ is the number of positions where $u_{K}$ and $v_{K}$ are identical, $k-d$ is the number of positions where $u_{K}$ and $v_{K}$ are distinct. When $d$ is the number of positions where $u_{K}$ and $v_{K}$ are distinct, $k-d$ is the number of positions where $u_{K}$ and $v_{K}$ are identical. In any case, $k-2 d=\left|\left\langle u_{K}, v_{K}\right\rangle\right|$. So, $d=\left(k-\left|\left\langle u_{K}, v_{K}\right\rangle\right|\right) / 2$. In $B_{a}\left(H^{K}\right), a^{i}$ and $a^{k-i}$ can be replaced by $a^{\left(k-\left|\left\langle u_{K}, v_{K}\right\rangle\right|\right) / 2}$ and $a^{\left(k+\left|\left\langle u_{K}, v_{K}\right\rangle\right|\right) / 2}$, respectively. This connection may reduce the computation since the inner product is easier to compute than the symmetric distance $d$.

\section{Classification of Hadamard matrices of order 24}

In this section, to show the sensitivity of our new algorithm proposed in the last section, we apply it to classify the known 60 Hadamard matrices of order 24 . We downloaded these matrices from http://www.research.att.com/ njas/hadamard/ and we order them as they were ordered on the web page. To save space, we do not list the detailed numerical results that are put into Tables 1 and 2, which can be found in our technical report [5], which appeared on the web site http://www.math. hkbu.edu.hk as an appendix to the paper.

Take $a=\pi \approx 3.1415926$; we first compute $F_{B_{a, 4}}(H)$ for these 60 Hadamard matrices. The computation results are listed in the 5 left columns of Table 1 (see [5]), where we just report the integer part for each $B_{a}$-value (denoted by $\left[B_{a}\right]$ ) and their frequencies. From the above computation results, we can see that there are at least 35 inequivalent classes for Hadamard matrices of order 24. Furthermore, we compute $F_{B_{a, 6}}(H)$ for the 38 unseparated Hadamard matrices. To save space, we only report the frequency of $\left[B_{a, 6}\right]=34953$ for all the 38 matrices in the last column of Table 1. From Table 1, we can see that the 60 Hadamard matrices are pairwise inequivalent.

From the above results, we can see that our algorithm is more sensitive than that of the profiles. In fact, as pointed out in [16], for Hadamard matrices of order 24 there are 35 classes for 4-profiles; 45 classes for 8-profiles; 42 classes for extended 4 -profiles; and 48 classes for generalized 4 -profiles. Neither one can distinguish the 60 classes. 


\section{Classifichtion of Hadamard matrices of order 36}

In this section, as a demonstration of applications of our new algorithm to classification of Hadamard matrices, we apply again the algorithm proposed in Section 3 to classify the known 192 Hadamard matrices of order 36. They were downloaded from the web site http://www.uow.edu.au/ jennie/matrices/. The first 180 Hadamard matrices were supplied by E. Spence (see [21]). The next 11 Hadamard matrices were supplied by V. D. Tonchev (see [22]). The last one was given by Z. Janko. In the following, we shall adopt the corresponding sequence orders as they were ordered on the web page to denote these 192 Hadamard matrices. We shall also use $1000+i$ to represent the transpose of the $i$-th Hadamard matrix for $1 \leq i \leq 192$. Note that $H_{i}{ }^{\prime}=H_{i}$ for $i=1$ and 174 . That means there are 382 distinct Hadamard matrices of order 36 in this study. All these Hadamard matrices have been put on the web site http://www.math.hkbu.edu.hk as an appendix to the paper.

Taking $a=\pi \approx 3.1415926$, we first compute $F_{B_{a, 4}}(H)$ for the 382 Hadamard matrices. The computation results are listed in the 6 left columns of Table 2 (see [5]), where we just report the integer part for each $B_{a}$-value and their frequencies. From the above computation results, we can see that there are at least 269 inequivalent classes for Hadamard matrices of order 36. Furthermore, we compute $F_{B_{a, 6}}(H)$ for the 204 unseparated Hadamard matrices. Here, we only report the frequency of $\left[B_{a, 6}\right]=86610$ for all the 204 matrices, which is listed in the last column of Table 2. Up to now, we can see that the 382 Hadamard matrices are pairwise inequivalent except possibly the pairs $(160,1160)$ and $(191,1191)$. Note that the frequency of $\left[B_{a, 6}\right]=87814$ for matrix 160 and 1160 is 0 and 3, respectively. Similarly, the frequency of $\left[B_{a, 6}\right]=88389$ for matrix 191 and 1191 is 3971 and 4214 , respectively. Hence, we can announce that there are at least 382 inequivalent classes for Hadamard matrices of order 36. It seems to us that this discovery is new.

\section{DiscusSiOn AND CONCLUDING REMARKS}

In this paper we propose some new criteria for equivalence of Hadamard matrices and a related algorithm. We apply this algorithm to classify the known 60 inequivalent Hadamard matrices of order 24 and the 192 Hadamard matrices of order 36 and their transposes. It turns out that we can easily find that they are pairwise inequivalent. The new algorithm as well as the profiles are an attempt to provide a canonical form to sorts. To classify $m$ Hadamard matrices, it is not necessary to test all pairs, a procedure requiring $O\left(\mathrm{~m}^{2}\right)$ comparisons between two matrices. In the new algorithm, each matrix is tested and related information (parameters) are tabulated. Then the tabulated values can be compared. This is an advantage of the new algorithm. Additionally, from the computation process, we found out that $F_{B_{a, 2 i+1}}(H)$ has almost the same effect as that of $F_{B_{a, 2 i}}(H)$ to distinguish the inequivalence of Hadamard matrices. So we can modify Steps 1 and 3 of the algorithm as follows:

Step 1. Let $k=4$.

Step 3. If $k=n / 2$, we fail to identify equivalence between $H_{1}$ and $H_{2}$ and need further study; otherwise let $k:=k+2$ and go to Step 2 .

Although the algorithm complexity to compute $F_{B_{a, k}}(H)\left(O\left(k n^{2}\left(\begin{array}{c}n \\ k\end{array}\right)\right)\right)$ is in general a little bit larger than that to compute $k$-profiles $\left(O\left(k n\left(\begin{array}{c}n \\ k\end{array}\right)\right)\right)$ for any given Hadamard matrix $H$ of order $n$, the complete classification of Hadamard matrices 
of order 24 shows that the new algorithm is significantly faster on average and more sensitive than the profiles. In fact, we have also applied the 4-profiles to these 382 Hadamard matrices of order 36 and found that the profile cannot distinguish each matrix and its transpose matrix for these 192 Hadamard matrices. The situation is even worse - the profile cannot detect pairwise inequivalence for all these 192 matrices. It can detect only 173 pairwise inequivalent classes from these 192 matrices by the 4-profiles. This shows again that the new algorithm is more sensitive than the profiles.

The algorithm proposed in this paper is efficient only for detecting inequivalence of Hadamard matrices. It cannot identify two equivalent Hadamard matrices. For two equivalent Hadamard matrices the new algorithm cannot give any conclusion after we carry out $n / 2-2$ tests or $n / 4-1$ tests if you use the modified algorithm. Therefore, the algorithm is only a necessary algorithm, like Smith normal form and profiles. For identifying equivalence of two Hadamard matrices, we need some efficient algorithm. A complete search is tractable only for small order $n$, for example, $n \leq 28$ (see [12], [13], 20]). A further study is needed.

The criterion $B_{a}(H)$ and its projection $B_{a, k}(H)$ have a close relationship with measures of uniformity in quasi-Monte Carlo methods. For example, the centered $L_{2}$-discrepancy $(\mathrm{CD})$ and warp-around $L_{2}$-discrepancy (WD) proposed by Hickernell (1998) can measure uniformity of a set of points on a unit cube. Let $\mathcal{P}=\left\{u_{1}, \ldots, u_{n}\right\}$ be a set of points on $C^{s}=[0,1]^{s}$. Its $\mathrm{CD}$ can be calculated by

$$
\begin{aligned}
& (C D(\mathcal{P}))^{2}=\left(\frac{13}{12}\right)^{s}-\frac{2}{n} \sum_{k=1}^{n} \prod_{j=1}^{s}\left(1+\frac{1}{2}\left|u_{k j}-0.5\right|-\frac{1}{2}\left|u_{k j}-0.5\right|^{2}\right) \\
& +\frac{1}{n^{2}} \sum_{k=1}^{n} \sum_{j=1}^{n} \prod_{i=1}^{s}\left[1+\frac{1}{2}\left|u_{k i}-0.5\right|+\frac{1}{2}\left|u_{j i}-0.5\right|-\frac{1}{2}\left|u_{k i}-u_{j i}\right|\right]
\end{aligned}
$$

where $u_{k}=\left(u_{k 1}, \ldots, u_{k s}\right)^{\prime}$. For any Hadamard matrix $H=\left(h_{i j}\right)$ of order $n$, its $n$ rows form a set of $n$ points over $C^{n}$, denoted by $\mathcal{P}_{H}$ if two entries -1 and 1 are mapped to $[0,1]$ by a linear transformation. Without loss of any generality we can choose $1 / 4$ and $3 / 4$ as two entries. We define the centered $L_{2}$-discrepancy of $H$, denoted by $C D(H)$, as the $\mathrm{CD}$ of $\mathcal{P}_{H}$.

$$
C D^{2}(H)=\left(\frac{13}{12}\right)^{n}-2\left(\frac{35}{32}\right)^{n}+\frac{1}{n^{2}}\left(\frac{5}{4}\right)^{n}\left(n+2 \sum_{i=1}^{n} \sum_{j=1}^{i-1}\left(\frac{4}{5}\right)^{d_{i j}}\right),
$$

where $d_{i j}$ is the Hamming distance between the $i$-th and $j$-th rows of $H$. As a sequence we have

$$
C D^{2}(H)=\left(\frac{13}{12}\right)^{n}-2\left(\frac{35}{32}\right)^{n}+\frac{1}{n^{2}}\left(\frac{5}{4}\right)^{n} \sum_{i=0}^{n} E_{i}(H)\left(\frac{4}{5}\right)^{i}
$$

which shows $C D^{2}(H)$ is a linear function of $\sum_{i=1}^{n} E_{i}(H)(4 / 5)^{i}$, where $E_{i}(H)$ is the number of pairs of two rows of $H$ whose Hamming distance is $i$. As $C D(H)$ is invariant only to permutations of rows, columns and negations on the columns, we cannot directly use it as a criterion in classification of Hadamard matrices. From formula (6.1), it is suggested that we choose $a=4 / 5$ in $B_{a}(H)$ and $B_{a, k}$. Unfortunately, the $B_{4 / 5, k}, k=3,4, \ldots, n / 2$, have less efficiency than the $B_{\pi, k}$. In fact, by computing the distribution of $B_{4 / 5, k}$ for $k=3,4,5,6$, we can only obtain 
368 inequivalent classes from these 382 Hadamard matrices. It cannot distinguish each of the 14 Hadamard matrices labelled 26, 35, 106, 137, 138, 163, 169, 175, 176, $177,179,180,187$ and 188 and their transposes. We believe that the parameter $a$ can be chosen as any small irrational number. A similar discussion can be applied to the warp-around $L_{2}$-discrepancy.

\section{ACKNOWLEDGMENTS}

The authors are grateful to the referee for his helpful comments and to Professor E. Spence and Professor J. Seberry for providing a lot of useful information by personal communication. A portion of this research was carried out while the second author was visiting Hong Kong Baptist University in 2001, and he wishes to express many thanks to the Department of Mathematics for its hospitality and services. The authors also thank Professor Fred J. Hickernell, Dr. Chang-Xing Ma and Professor L. Zhu for their valuable comments.

\section{REFERENCES}

[1] J. B. Clark and A. M. Dean, Equivalence of fractional factorial designs, Statistica Sinica 11 (2001), 537-547.

[2] C. J. Colbourn and J. H. Dinitz, CRC Handbook of Combinatorial Designs, CRC Press Inc., Boca Raton, 1996. MR 97a:05001

[3] J. Cooper, J. Milas and W. D. Wallis, Hadamard equivalence, Comb. Math., Proc. Int. Conf., Canberra 1977, Lect. Notes Math. 686 (1978), 126-135. MR 80c:05043

[4] J. G. Dumas, B. D. Saunders and G. Villard, On efficient sparse integer matrix Smith normal form computations, J. Symb. Comput. 32 (2001), 71-100. MR 2002d:65042

[5] K. T. Fang and G. Ge, An efficient algorithm for the classification of Hadamard matrices, Technical Report MATH-298, Hong Kong Baptist University, 2001.

[6] S. Georgiou, C. Koukouvinos, M. Mitrouli and J. Seberry, Necessary and sufficient conditions for three and four variable orthogonal designs in order 36, J. Statist. Plann. Inference 106 (2002), 329-352. MR 2003g:62132

[7] M. Hall, Jr., Hadamard matrices of order 16, J.P.L. Research Summary 36-10, 1 (1961), $21-26$.

[8] M. Hall, Jr., Hadamard matrices of order 20, J.P.L. Technical Report, 32-761, 1965.

[9] F. J. Hickernell, "Lattice rules: how well do they measure up?" in Random and Quasi-Random Point Sets, Eds P. Hellekalek and G. Larcher, Springer-Verlag, 106-166, 1998.

[10] N. Ito, J. S. Leon and J. Q. Longyear, Classification of 3-(24,12,5) designs and 24-dimensional Hadamard matrices, J. Combin. Theory Ser. A 27 (1979), 289-306.

[11] H. Kimura, New Hadamard matrices of order 24, Graphs Combin. 5 (1989), 236-242.

[12] H. Kimura, Classification of Hadamard matrices of order 28 with Hall sets, Discrete Math. 128 (1994), 257-268. MR 94m:05046

[13] H. Kimura, Classification of Hadamard matrices of order 28, Discrete Math. 133 (1994), 171-180. MR 95i:05040

[14] H. Kimura and H. Ohmori, Construction of Hadamard matrices of order 28, Graphs Combin. 2 (1986), 247-257. MR 89h:05019

[15] C. Lin, W. D. Wallis and L. Zhu, Extended 4-profiles of Hadamard matrices, Ann. Discrete Math. 51 (1992), 175-180.

[16] C. Lin, W. D. Wallis and L. Zhu, Generalized 4-profiles of Hadamard matrices, J. Comb. Inf. Syst. Sci. 18 (1993), 397-400. MR 96a:05032

[17] C. Lin, W. D. Wallis and L. Zhu, Equivalence classes of Hadamard matrices of order 32, Congr. Numerantium 95 (1993), 179-182. MR 94m:05047

[18] C. X. Ma, K. T. Fang and D. K. J. Lin, On isomorphism of fractional factorial designs, J. Complexity 17 (2001), 86-97. MR 2002b:05021

[19] H. Niederreiter, Random Number Generation and Quasi-Monte Carlo Methods, SIAM CBMS-NSF Regional Conference Series in Applied Mathematics, Philadelphia, 1992. MR 93h:65008 
[20] E. Spence, Classification of Hadamard matrices of order 24 and 28, Discrete Math. 140 (1995), 185-243. MR 96b:05040

[21] E. Spence, Regular two-graphs on 36 vertices, Lin. Alg. and its App. 226-228 (1995), 459497. MR 96c:05167

[22] V. D. Tonchev, Hadamard matrices of order 36 with automorphism of order 17, Nagoya Math. J. 104 (1986), 163-174. MR 88a:05032

Department of Mathematics, Hong Kong Baptist University, Kowloon Tong, Hong Kong, CHINA

E-mail address: ktfang@math.hkbu.edu.hk

Department of Mathematics, Suzhou University, Suzhou, 215006, China

E-mail address: gnge@public1.sz.js.cn

Current address: Department of Mathematics, Zhejiang University, Hongzhou 310027, Zhejiang, China 